\title{
Sustainable enterprise interoperability from the Activity Domain Theory perspective
}

\author{
Lars Taxén
}

\section{Linköping University Post Print}

\section{Tweet}

N.B.: When citing this work, cite the original article.

Original Publication:

Lars Taxén, Sustainable enterprise interoperability from the Activity Domain Theory perspective, 2012, Computers in industry (Print), (63), 8, 835-843.

http://dx.doi.org/10.1016/j.compind.2012.08.011

Copyright: Elsevier

http://www.elsevier.com/

Postprint available at: Linköping University Electronic Press

http://urn.kb.se/resolve?urn=urn:nbn:se:liu:diva-86658 


\title{
Sustainable Enterprise Interoperability from the Activity Domain Theory perspective
}

Lars Taxén,

Linköping University

Post mail: Rundan 91

SE-14645 Tullinge, Sweden

Tel: $\quad$ +46-073-0977864

E-mail: $\quad$ lars.taxen@telia.com

\begin{abstract}
The investigation of sustainable enterprise interoperability requires an explicit position of what constitutes the enterprise. In particular, human aspects like agency, sense-making, interpretation, common understanding, etc., must be taken into account. To this end, the purpose of this contribution is to make an inquiry into interoperability from the perspective of the Activity Domain Theory (ADT). In this theory, the enterprise is considered as a constellation of activity domains. The activity domain is characterized by the activity modalities - objectivation, contextualization, spatialization, temporalization stabilization, transition - which represent human, innate predispositions for coordinating actions. Interoperability is focused to one particular modality: the transition between activity domains. An example from the telecom industry is used to illustrate the approach. Implications for various aspects of interoperability are discussed. In conclusion, it is argued that the ADT is an alternative framework for advancing the understanding of sustainable interoperability between enterprises and enterprise systems.
\end{abstract}

Keywords: Interoperability, enterprise, enterprise systems, ERP, PLM, Activity Domain Theory, Activity Modalities, socially organized work.

\section{INTRODUCTION}

The traditional, vertical integration of enterprises, where a single enterprise controls the bulk part of the supply chain, is rapidly being complemented by close and volatile collaborations between independent organizations. In order to capture shortterm business opportunities, there is a need to establish and maintain sustainable networks of enterprises that benefit all partners involved. Sustainability - the capacity to endure - implies, among other things, the discovery of new and lost enterprise capabilities in the network, and the adaption of knowledge, processes and interacting enterprise systems (ES) to the new circumstances. This trend brings forward an increased focus on enterprise interoperability. Whatever change impacts the network, it will inevitably affect interoperability. Thus, in order to achieve sustainability, it is imperative to understand the essence of interoperability.

Interoperability was originally defined as "the ability of two or more systems or components to exchange information and to use the information that has been exchanged" (IEEE, 1990). However, in the context of sustainability, this definition is clearly inadequate. The scope must be widened from a narrow focus on ESs to encompass the interoperating entities - the enterprises. A firm position must be taken on what constitutes the enterprise. Unless such a position is defined, inquiries into interoperability will inevitably be based on a morass underpinning, which aggra- 
vates a cumulative knowledge build up and the elaboration of realistic approaches for intervening in practice.

The concept of 'enterprise' is closely related to 'organization', where 'enterprise' emphasizes the doing or undertaking aspects of the business, and 'organization' the more structural ones. However, there is no consensus in the literature about what constitutes an organization. A number of different Unit of Analysis (UoA) have been suggested, such as: "organization" (Levinthal \& Warglien, 1999), "individual act" (Morgeson \& Hofmann, 1999), "dyad" (Sosa, 2011), "organizational field" (Schoonhoven, Meyer, \& Walsh, 2005), "practice" (Brown \& Duguid, 1991), "organizational routines" (Volkoff, Strong, \& Elmes, 2007), "transaction" (Argyres, 1999), "activity" (Nickerson \& Zenger, 2002), "social actor" (King, Felin, \& Whetten, 2010), "work teams" (Nonaka \& von Krogh, 2009), and "work system" (Alter, 2006).

These different UoAs focus each on a particular aspect of the organisation, leaving the core character of the organization in oblivion. Thus, in order to put interoperability inquires on firm ground, a conceptualization of what constitutes the fundament of the organization is needed. Such a fundament is provided by Activity Domain Theory (ADT; Taxén, 2009).

The ADT grew over many years from two roots - a theoretical one in the Russian theory of Activity (Kaptelinin, \& Nardi, 2006) and a practical one in the Ericsson telecom practice, where the author spent most of his professional life ${ }^{1}$. A strong motivation for engaging in ADT is that most extant approaches tend to overlook human, biological inherited prerequisites for acting. This is indeed remarkable, since every act must ultimately be grounded in our biological and cognitive human constitution; we cannot act outside our given prerequisites.

In order to address this issue, ADT is centred on the concept of coordination. Coordination is at the core of human reality; by necessity we need to coordinate our actions, both when acting alone and together with other people. Coordination has been studied extensively in the literature; however, mainly from an organizational point of view. For example, Grant claims that the "fundamental task of the organization is to coordinate the efforts of many specialists. Although widely addressed, organization theory lacks a rigorous integrated, well developed and widely agreed theory of coordination." (Grant, 1996, p. 113). Concerning a particular kind of ESs, the so called Enterprise Resource Planning (ERP) systems, Kim, Lee, \& Gosain state that "The problem of coordination is counted as one of the most important issues leading to failure of a number of ERP implementations" (Kim, Lee, \& Gosain, 2005, p. 159).

In spite of the extensive amount of research, it is remarkably hard to pin down coordination. For example, Larsson (1990) lists nineteen definitions, and Malone \& Crowston (1994) identify eleven interpretations. Malone \& Crowston also emphasize the multidisciplinary nature of coordination; the study of coordination must draw on organisation theory, management science, computer science, economics, linguistics, and psychology (ibid, p. 88). To further aggravate this situation, Nicolini concludes that there is a lack of knowledge about how coordination is actually carried out in practice:

\footnotetext{
${ }^{1}$ Ericsson is a well-known leading supplier of telecommunication equipments worldwide: http://www.ericsson.com/
} 
In spite of the recent resurgence of interest in the study of coordination (Bechky 2003, 2006), we still know markedly little about the practice of coordination and, above all, the coordination of practices and knowings. (Nicolini, 2011, p. 617)

In order to illustrate the approach to coordination suggested by ADT, we may consider a familiar activity - that of making a cup of coffee at breakfast. First, there is certainly a motive for doing so: enjoying a good taste, becoming alert, and so on. The activity is directed towards an object: the coffee that eventually will appear in the cup - an "'objectless' activity is impossible" (Kaptelinin \& Nardi, 2006, p. 55). By attending the object, motivated by some need, we contextualize the situation at hand $-\mathrm{a}$ 'horizon of relevance' is constructed that enables us to concentrate on what is appropriate for the task at hand, and disregard irrelevant aspects. In the coffeemaking situation, this context probably includes the kitchen, the package of coffee, the coffee urn, etc., while other things like the uncut lawn outside the kitchen window and the weather are more or less irrelevant for the moment.

When a context has been perceived, there is a need to orient oneself in the situation: how are the relevant things related to each other? Where is the package of coffee? Where is the outlet for the cord to the coffee urn? Etc. If you have been making coffee in the same kitchen for a long time, this is probably not an issue; you know where to find the things you need and how these are located in relation to each other. However, if you come into quite a new kitchen, you have to make a conscious effort to orient yourself in that situation.

Next, you have to perform a sequence of actions in order to get the desired coffee: get hold of the coffee package, load the right amount of coffee into the coffee urn, switch on the power button, pour the coffee when it is ready, and the like. You have learnt this sequence, either by yourself through a process of trial and error, or, which is more likely, by someone showing you how. This lends a stabilizing character to the situation; you do not have to start all over again every morning by evaluating all possible ways of making good coffee.

During the entire activity you make use of certain means without which the activity could not be performed: coffee beans, the coffee urn, electricity outlets, the cord between the outlet and the coffee urn, cups, chairs to sit on, possibly a table to put the cup on, and the like. Not all properties of these means are interesting; only those that are relevant in the coffee-brewing situation.

Finally, after enjoying your well-earned cup of coffee, you may turn your attention to something else, like starting your trip to work, cleaning the house, washing your car or whatever. In doing so, you need to make turn your attention to another activity, where you will encounter the same characteristics as in the coffee-making activity; this time however, manifested in a different way according to the new situation.

In $\mathrm{ADT}$, the fundamental conjecture is that the characteristic features described above are found in every socially organized human activity. Coordination is enabled by mastering the dimensions spanned by the activity modalities - objectivation, contextualization, spatialization, temporalization, stabilization and transition. These are integrated into a totality of the situation called the activity domain.

When applying this thinking to enterprises, it can be observed that an enterprise consists of many organized groups of people working on different objects for various reasons. For example, a marketing business unit sells the product, development develops it, and production produces it. Thus, an enterprise can be conceptualized as a constellation of activity domains, each providing a certain capability that the en- 
terprise needs. Regardless of what kind of organizational unit is considered - two collaborating persons, a team, a group, a project, a business unit, the entire organization, collaborating organizations, and so on, - these may all be considered activity domains, each one structured by its specific manifestations of the activity modalities.

In order to carry out the work in an activity domain, different kinds of means such as ESs are needed. These systems provide information management capabilities, the relevance of which in a particular domain depends on its object and motive. For example, an ERP system is likely to be more relevant in domains that sell commodities from stock than in a domain that develops software functionality.

In this perspective, interoperability is seen as interoperability between activity domains. Stated differently, interoperability occurs between different contexts, indicating that the transition modality is in focus. The transition may affect such disparate things as agreeing on what a certain information entity means, deciding a sequence of information transfer, providing data base interaction facilities between ESs, securing stable Intranet services, and the like.

With this as a background, the paper is structured as follows. To begin with, the main features of the activity domain are outlined. Next, various aspects of interoperability are analyzed with the help of an example from Ericsson. This is followed by a discussion of the implications of the ADT approach towards interoperability. Since the contribution is a conceptual paper, no fully completed case is provided. However, the implications are derived from solid experiences learnt in the practice of developing complex telecommunication systems for an extremely demanding market, which makes it plausible that the approach is widely applicable. In conclusion, I claim that the proposed approach provides a firm ground for investigating sustainable interoperability and devising methods and means to manage it in practice.

\section{THE ACTIVITY DOMAIN}

In Figure 1 the activity of performing a guitar concert is illustrated:

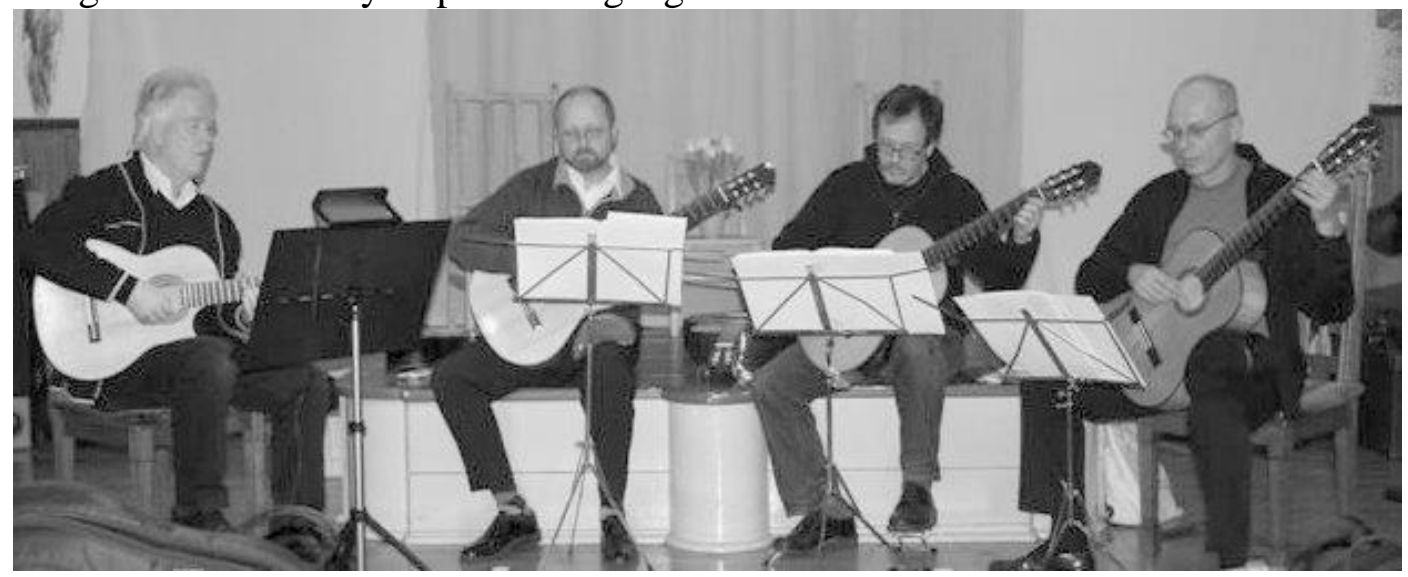

Figure 1: A guitar quartet in concert

The motive for engaging in this activity may be to amuse an audience, play for money, personal satisfaction, and the like. The object in focus for the activity is evident - the music to be played in the concert. Together, the motive and the object make up an "attraction point" around which a context of relevance for the activity is formed. 
A very basic condition for giving a concert is of course that the players can play on their instruments. Learning to play a guitar means a long and arduous practice in which the player learns how to coordinate left and right hands, how to read notes, how to uphold an efficient sitting position, and much more. As time goes by, the player and his guitar may eventually become so interweaved that they form a unity the player becomes "one" with his instrument, as illustrated by the following quotation from the cellist Mstislav Rostropovich:

There no longer exist relations between us. Some time ago I lost my sense of the border between us.... I experience no difficulty in playing sounds... The cello is my tool no more.

(Zinchenko, 1996, p. 295)

This means that it is the joint capabilities of the player and the instrument that enables the activity. Together, they constitute what in Activity Theory is called a 'functional organ' (Kaptelinin \& Nardi, 2006). If the player cannot play, or if the instrument is unable to produce enjoyable sounds, the activity cannot be carried out.

Another obvious condition is that the players have to coordinate the playing between themselves. In order to do so certain means such as the score in Figure 2 may be used:

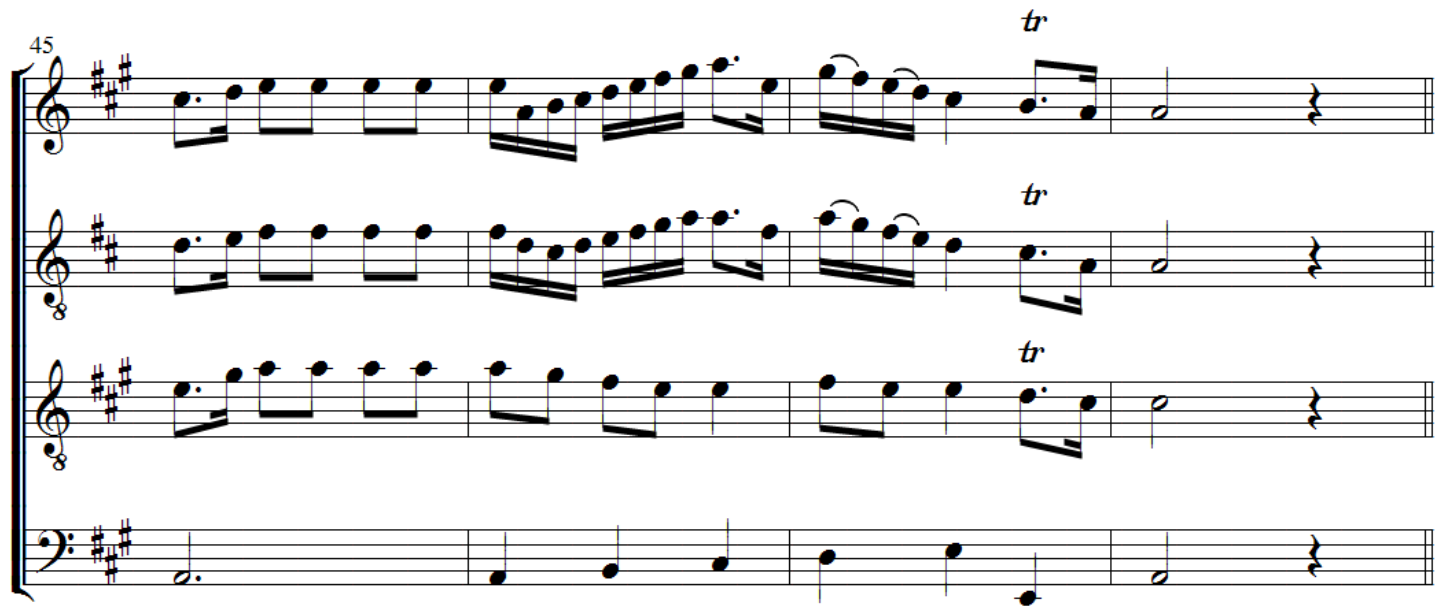

Figure 2: An example of a musical score

The particular form and structure of the score have evolved since the $14^{\text {th }}$ century when spacing and timing were brought together in the sheet music (Hoskin, 2004). It is quite likely that this form has adapted over the centuries to match the neural organization of our brain.

If we take a closer look at the score, it can be seen that it exhibit certain features. First, there is an obvious temporal dimension in the sequence of notes from left to right. Each note signifies a certain time interval as indicated by the stems and dots. Correspondingly, the vertical positions of the notes indicate a spatial dimension between the notes. This applies both to individual voices, where each note is positioned in relation to the note lines, as well as the position of the voices on top of each other.

Furthermore, notes cannot be played according to the preference of each individual player. The various signs in the score - the $m f$ indicating mezzo forte, the ?: symbolizing the F-clef, the \# showing that the key is A-flat, etc. - are social codes that must be adhered to when playing. This means that certain actions are valid, while others are not. Thus, the codes have a stabilizing function in the activity; if they are not acknowledged, the activity will disintegrate. 
Before anything can be played at all, the guitars must be built by a guitar maker. This is done in a quite different activity where the object is the guitar (see Figure 3):

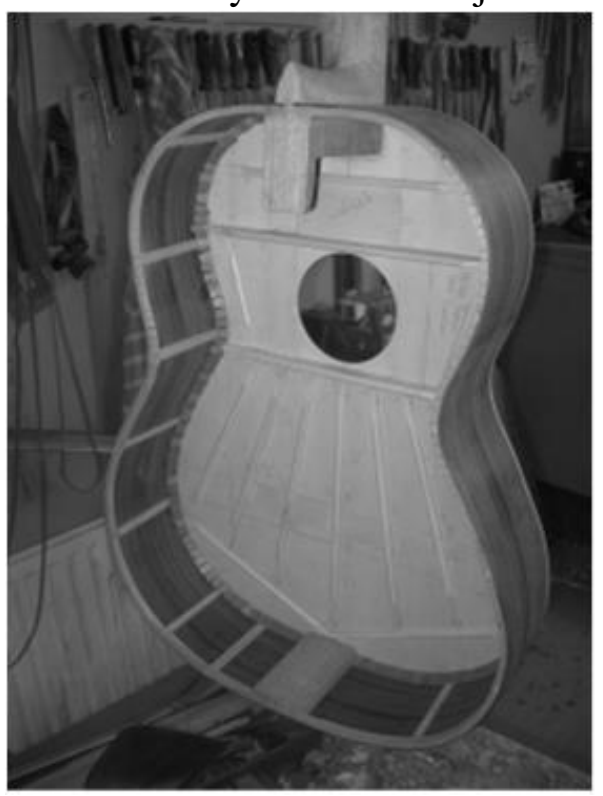

Figure 3: Building a guitar

In order for a guitar to be playable, certain aspects must be worked out in the transition between playing and guitar-making activities. This concerns things like the distance between the frets, the string tensions, the size of the guitar, and much more. However, there is no need for players and guitar makers to agree upon all details in each their respective activity (in fact, this is not even possible).

The features outlined above are, according to ADT, universal features; they can be found in every socially organized human activity:

- Human activity is always motivated by some need and directed towards an object (objectivation).

- The object and motive frame the context in which actions make sense (contextualization).

- Acting requires a spatial comprehension of the context (spatialization).

- Actions are carried out in a certain order (temporalization)

- The activity is stabilized by adhering to rules, norms, etc., that signify which actions are valid (stabilization).

- When activities with different motives and objects interact, the transition between these activities has to be sorted out (transition).

- Combinations of human capabilities and means capabilities are used to carry out actions.

- Everything relevant in the activity domain - the motive and object; the actors; tools; language, activities, things, rules, norms, etc. are all integrated into a meaningful whole. This means that every domain will construct a world of its own; parts of which have to be reconciled with the "world views" of other domains if collaboration between domains shall be possible. 
As stated above, the conceptualization of activity in this way is called the activity domain. The different dimensions of objectivation, contextualization, spatialization, temporalization, stabilization, and transition are called activity modalities. This concept alludes to "sensory modalities"; indicating that sensory impressions arriving in various sensory modalities are integrated into an action 'field' characterized by the activity modalities.

The claim that the activity domain and the activity modalities are universal is grounded in their phylogenetic origin, i.e., art-specific evolution of the human species. At birth, we are endowed with certain faculties for coordinating actions. These faculties are manifested differently during the ontogenetic, i.e., individual-specific development, depending on the particular circumstances that the individual encounters during her lifetime (Taxén, 2011).

\section{INTEROPERABILITY}

In order to illustrate how the ADT may inform interoperability, an example from Ericsson may be used. In Figure 4 below, a business process in Business Process Modelling Notation (BPMN) is illustrated.

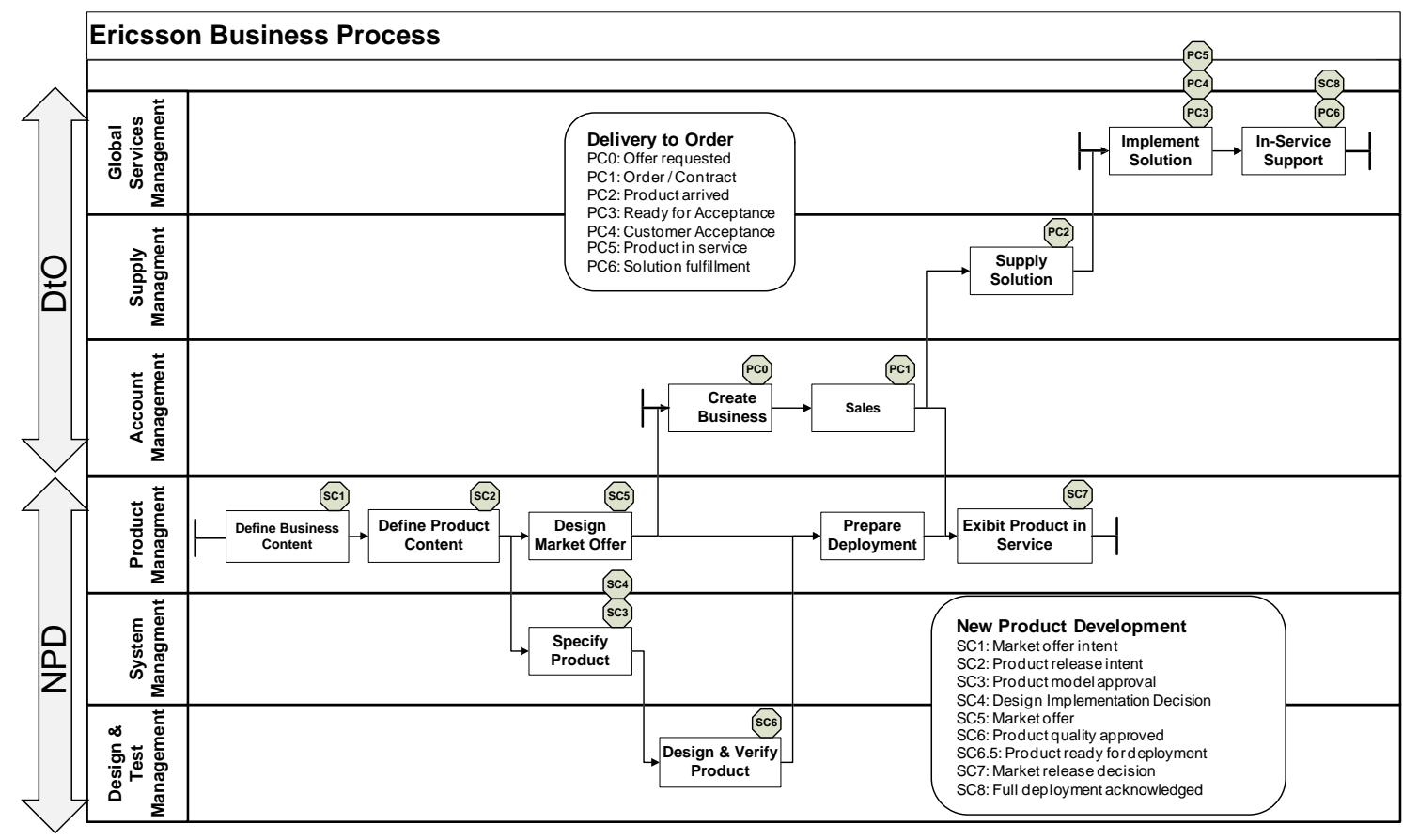

Figure 4: An example of a business process from Ericsson

Each swimlane (the horizontal lanes) represents a management area containing one or several activities. The swimlanes are grouped into 'Delivery to Order' (DtO), which sells systems that can be configured from existing modules, and 'New Product Development' (NPD), which develops new modules. The progress within each group is indicated by the ' $\mathrm{PC}$ ' and the SC' state sets respectively. Although this is an example of interoperating entities within an enterprise, the analysis that follow is valid for interoperating enterprises as well.

\section{The enterprise focus}

In ADT, the activities in each swimlane are regarded as activity domains; each one 
with its particular motive and object (henceforth called 'work object' for convenience). Everywhere a sequence flow (the lines between activities) crosses a swimlane border, a transition between domains is taking place (in fact, this is also valid for the transitions between domains in a swimlane as well). In Figure 5 a closeup of one of these transitions is illustrated:

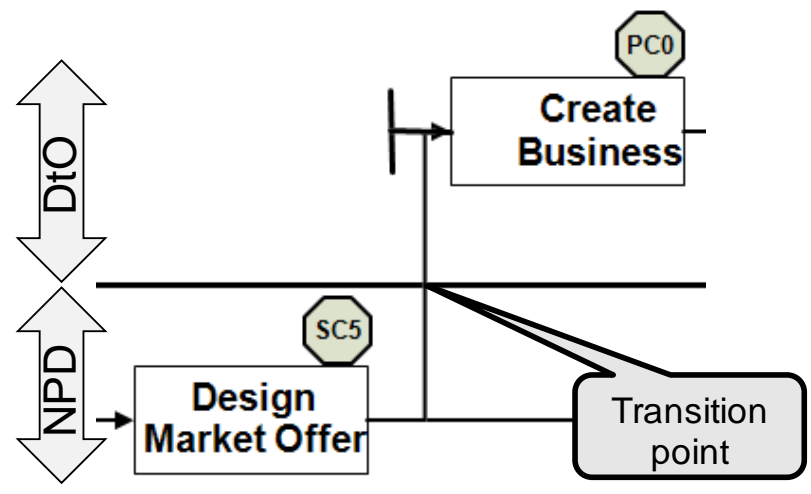

Figure 5: The transition between two activity domains

In each such transition point, a manifestation, i.e. the concrete expression of the transition modality must be elaborated. When defining transition, it must be kept in mind that all modalities are interdependent. It will not work to treat them as separate and independent. At least the following issues have to be considered.

\section{Activity domain characteristics}

To begin with, the motives and work objects for each domain involved must be attended. For 'Design Market Offer' this is "to create attractive market offerings, packaged and prepared for efficient marketing and sales". The work object is simply called 'Market Offer'. Similarly, the motive for 'Create business' is to "understand customer needs and establish relations with customers, and at the same time promote an understanding among customers of Ericsson's market offerings and the ways in which they can provide satisfaction". The work object for this domain is "Business Intent'.

The reason why this issue is important is that the motive and the work objects are, as stated above, the drivers of how each domain is constructed. If the motives and work objects are vague, this will inevitably 'spill over' onto the characterization of the domain, which in turn will impact the implementation of interoperability between ESs.

\section{Data exchange (spatialization)}

The next issue is to define the manifestation of the spatialization modality in terms of which things are important in the transition, and how these things are characterized and related to each other. Such things that need to be managed in an ES are usually called information entities, managed items, and the like.

In order to concretize this, we may examine the outcome from 'Design Market Offer', which consists of the following entities:

- General product sales information

- Sales product package structure

- Customer value and key sales arguments 
- Announcement material

- Pricing and configuration guide

- Training material for marketing and sales

- Customer guides for introduction, substitution or termination of product

- Promotion, presentation and advertising material

Together, these entities are part of the work object 'Market Offer', some of which become input to 'Create Business' and others to 'Prepare Deployment' (see Figure 4). In order to manage the entities in the ESs, they have to be defined in an information model with respect to aspects like identification, classifications, state sets, attributes, revision rules, and the like (see the example in Figure 6):

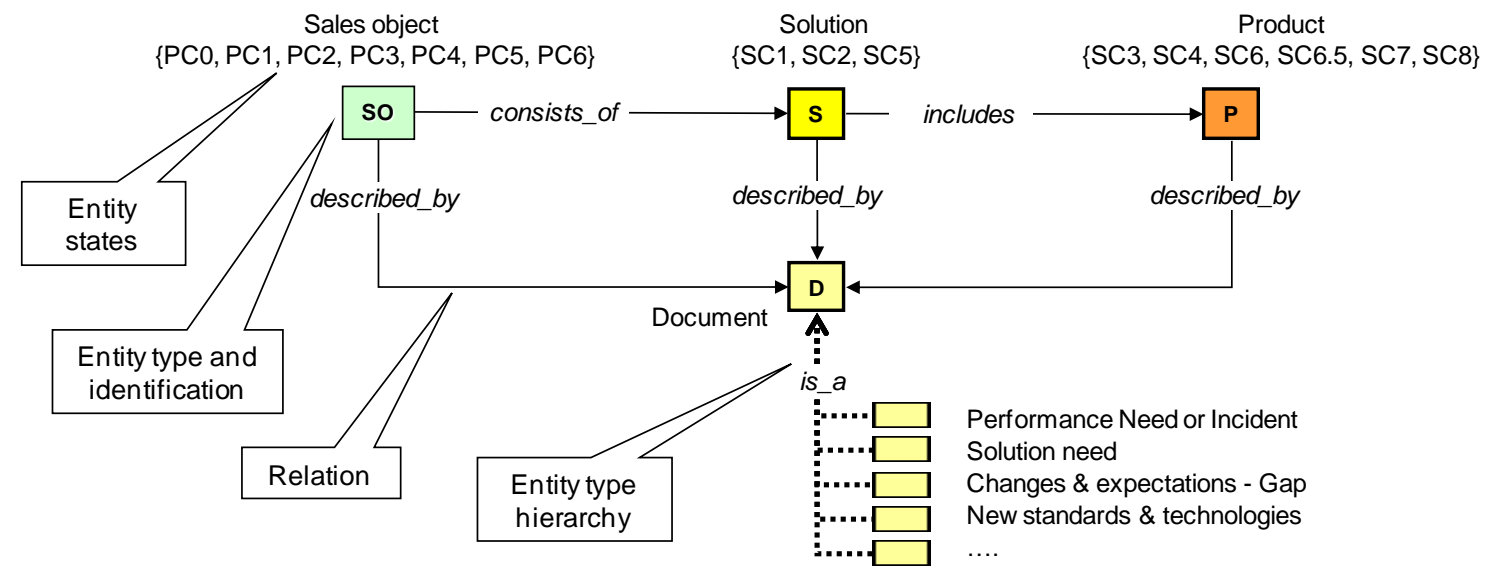

Figure 6: An example of an information model for the Ericsson activity domain

In this model, most of the entities from 'Design Market Offer' will probably be classified as documents.

The definition of the information model is a joint effort for actors in the domains 'Market Offer', 'Create Business', and that of the top domain itself - the Ericsson one. However, this is not enough. Since domains construct their own world-views, they will in general characterize internal entities in a different way. This implies that a transition between the internal and external characterization of a particular entity must also be elaborated.

Work flows (temporalization)

Another issue is to define the dynamics of the exchange between the ESs, i.e. the manifestation of the temporalization modality. This amounts to the specification of some kind of protocol between the ESs.

\section{Business rules (stabilization)}

Business rules, valid for the transition, need to be defined. Such rules, which is a manifestation of the stabilization modality, may concern access rights, i.e. who is authorized to start a transition, prerequisites for doing so, rules for translating state sets between domains, revision rules, and the like.

\section{Commonality}

The actors must come to a certain consensus about the meaning of whatever is 
involved in the transition between the domains. The effort of arriving at such a consensus is indeed an awesome task, something which is mostly disregarded in most modeling approaches today.

\section{The enterprise system focus}

Having established what it takes to perform the transition between activity domains, this transition needs to be supported by the capabilities that ESs offer. Returning to the example in Figure 4, the 'Delivery to Order' domains are usually supported by ERP (Enterprise Resource Planning) ESs, and 'New Product Development' by PLM (Product Lifecycle Management) $\mathrm{ESs}^{2}$. As indicated in Figure 7, there are two places where an information exchange is taken place between the ERP and the PLM systems:

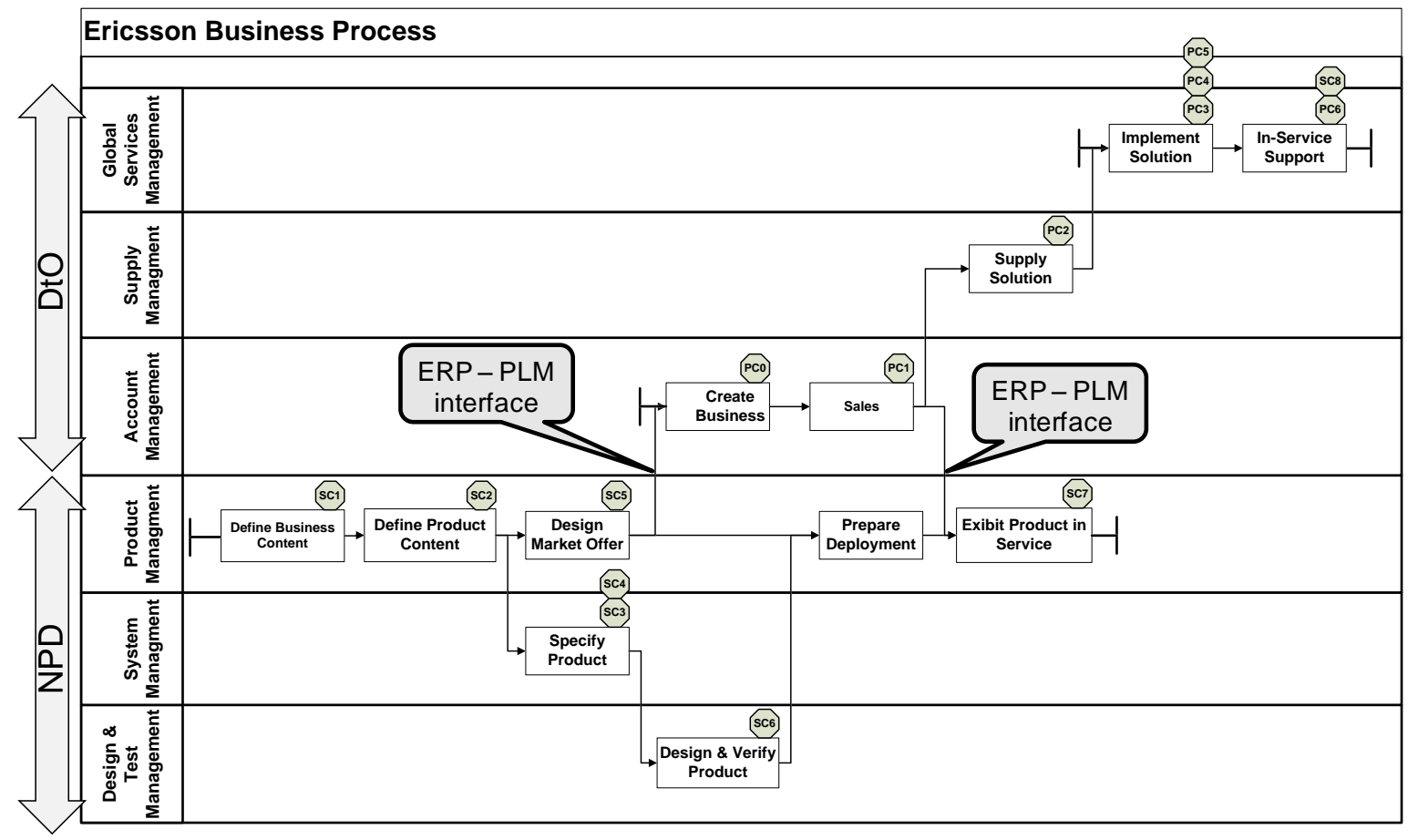

Figure 7: Transition points between ERP - PLM systems

A fact that complicates the implementation of interfaces between ERP and PLM systems is that they differ in some fundamental respects. ERP systems are transactional in nature, which means that they collect, store, modify, and retrieve the transactions of an organization. ${ }^{3}$ Moreover, ERP systems do not maintain revisions of a particular entity such as a product with all its associated documents. PLMsystems, on the other hand, are used to track different configurations and versions of a product or service during its life-time. This means that special measures must be

\footnotetext{
${ }^{2}$ As can be seen from Figure 7, the information managed in the PLM systems is a prerequisite for the information managed in the ERP system. However, in spite of their importance in product developing organizations, PLM systems are virtually non-existing in the research literature.

${ }^{3}$ A transaction is "an agreement, communication, or movement carried out between separate entities or objects, often involving the exchange of items of value, such as information, goods, services, and money" (Wikipedia, 2011)
} 
taken for, for example, tracing a delivery at a customer to a certain revision kept in the PLM system; something that is important for correcting errors found in the system.

In addition to the interfaces between two different kinds of ESs, each crossing of swimlanes within 'Delivery to Order' and 'New Product Development' also implies a transition, for example the transition between 'Define Product Content' and 'Specify Product'. Both these domains are supported by the PLM system. The same applies to the ERP system (see Figure 8):

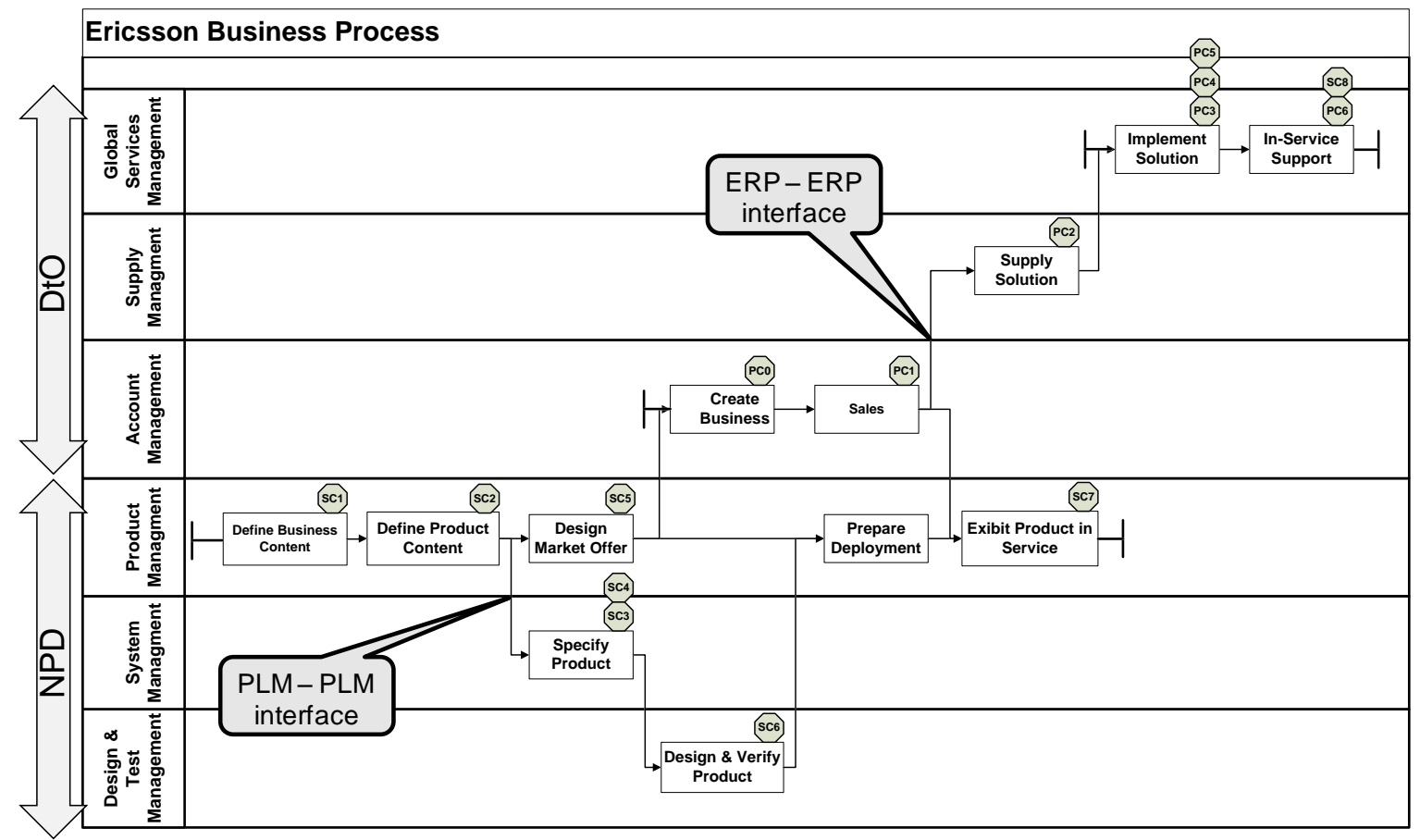

Figure 8: Transition points between same kinds of ESs

However, just because the same ES platform is used in both cases, the same transitional issues as for the ERP-PLM interface are valid also in this case. The implementation of the interface may of course be easier, since they are based on the same ES platform. ${ }^{4}$

\section{DISCUSSION}

The ADT approach brings about a number of implications for interoperability as follows.

The ontological foundation of the enterprise

The question of what constitutes the core of the organization is given a definite answer in ADT: the activity domain. This means that the same construct can be applied to any organizational 'level'. This in turn greatly alleviates the cognitive load of making sense about the organization, and consequently, the effort of implementing interoperability.

\footnotetext{
${ }^{4}$ The development and implementation of the ES applications and the interfaces is outside the scope of this paper. This issue has however been treated in Taxén (2011b).
} 
A choice between paradigms

In Figure 9, one of the activities in the Ericsson business process is illustrated. What difference does it make if we regard this activity as an element in a business process or as an activity domain?

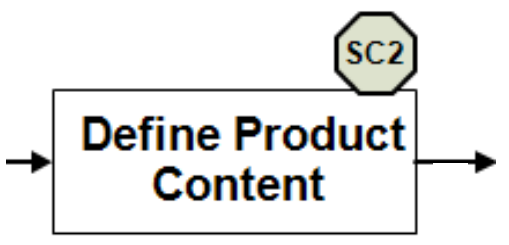

Figure 9: An activity

By regarding 'Define Product Content' as an activity domain, we see this activity as a situated, socially organized work context, constituted by its motive and work object. This context integrates everything needed to produce the outcome - human actors, tools, language, communication, symbols, signs, etc., as well as other activity domains needed. The integration also includes the coordination of actions, which is made possible by developing coordinative capabilities in all activity modalities, including the transition to other domains. This means that information models, processes, rules, as well as terms and concepts, are all internal to the activity domain; thus making the activity domain the central organizing construct.

If we, on the other hand, regard 'Define Product Content' as one activity in a sequence of steps in a business process, we place one of the modalities - temporalization - in focus. Other modalities will then become subordinated to the business process. This can be traced in sentences like "A Data Object represents information flowing through [my italics]"; "[Process modelling is the] capturing of an ordered sequence of business activities and supporting information [my italics] ${ }^{5}$.

On the other hand, other scholars claim that the information must be considered up front. For example, Kangassalo states that "Information systems should be defined on the basis of their conceptual content, not on the basis of the data flow" (Kangassalo, 1999, p. 105). In this case, the spatialization modality is in focus.

Whatever modality is emphasized, placing one of them as the central, organizing construct will not capture the full complexity of interoperability. This may severely aggravate implementation initiatives. If one modality only is attended, such initiatives will be based on a one-dimensional view of what is in fact a multidimensional complex. In particular, the achievement of commonality, and the interdependencies between the modalities may be neglected.

\section{Clarification of central concepts}

The way we think and talk about things will determine how we see the world, and consequently what actions we will find appropriate to take. This makes it important to scrutinize key concepts within the interoperability discourse. The definition of such concepts will be less opaque when using the activity domain as the UoA. Here are but a few examples:

\footnotetext{
${ }^{5}$ These examples are collected from the homepage of BPMN: http://www.bpmn.org/, but similar expressions are abundant in process modeling in general.
} 
- The concept of "Knowledge" is a quite tricky ("is knowledge embedded in artifacts?"; "can knowledge be treated as a commodity?"; "is knowledge what goes on between the ears?" and so on). In ADT, knowledge is always related to the work object and motive of the domain. This also implies that the concept of "capability" is to be preferred before "knowledge", since capability denotes an ability to do something, to act. Moreover, capability can be applied to both humans and whatever instruments used in carrying out actions.

- "Seamless" is a deceptive metaphor since it indicates a "boundary less" conceptualization. Taken to its extreme, if that happens (which it never will) the concept of "interoperability" becomes meaningless. In fact, the "seams" between domains are vital to acknowledge 6 . Any effort to tear down "context walls" is a dead end. Quite the opposite is necessary - if interoperability shall be possible to manage, relevant borders must be identified, defined and articulated.

- "Domain" is often used in different flavors, for example "application domain", "domain of discourse", and the like. In general, the "domain" concept is taken at face value without problemizing it. With the activity domain construct, the "domain" concept is given a precise interpretation.

- Often, models are referred to as being models of "the real world". Taken literally, this indicates that models are part of another world; the question is then: which world would that be? In ADT, models are considered means as real as any other means used in the elaboration of the activity domain. This issue may seem quite esoteric, but in fact, it affects the way we develop and employ models in implementation endeavors ${ }^{7}$.

\section{The role of Internet}

High expectations are placed on the future Internet as a universal business support system in which interoperability services can be invoked more or less on the fly according to various business needs. It is hoped that the Internet will empower enterprises to innovate by creating new business value in collaboration with other enterprises.

One possible enabler of such a collaboration is the Service-Oriented Architecture (SOA). In SOA, the terms "orchestration" and "choreography" are commonly used. Although there is (as usual) no consensus about the precise definition of these terms, it appears that "orchestration" stands for automated execution of workflows by some orchestration engine at runtime. Typically, such an orchestrated workflow is exposed as a service that can be invoked through an API. "Choreography" on the other hand, denotes an agreed-upon model of coordinated interactions between two or more parties in order to achieve a common goal.

From the ADT perspective, a service is a certain capability that someone needs in order to carry out some task. The service can be implemented in a different ways. For example, a PLM capability such as setting up a BOM (Bill of Material) can be provided by a PLM system on premises, or by a PLM system "in the Cloud". Although the technical delivery of the service indeed makes a difference, the important

\footnotetext{
${ }^{6}$ Just think about a "seamless" suite: this would probably be impossible to design and sell!

${ }^{7}$ This argument is elaborated in more detail in Taxén \& Lilliesköld (2008).
} 
issue is what it takes to set up and make operational such a service.

Stated differently, what is the character of the transition modality in a particular arrangement between a service provider and a service client? In order to automatically invoke a service available on the Internet, this service has to be standardized to the point that it is ES invariant, i.e., any ES can use it without modification. Since every enterprise is (and should be) unique in some way, this is not possible if taken to the extreme. A certain amount of adaption is always necessary, even if this can be quite minimal. Such services would probably fall under the category of "orchestration". When you move towards "choreography", services become progressively more difficult to "automatically" invoke, since such services put more focus on the entirety of the transition modality, for example the achievement of commonality between the parties.

Consequently, the invocation of interoperability services "on the fly" is not likely to come about but for the most highly standardized services, in which case they do not add to the core business of the ES. The specificity of activity domains involved, including very human aspects like interpretation, meaning, and the like, cannot be by-passed by technical advances such as SOA or the Cloud.

\section{Positioning the approach towards extant research}

The main contribution of the ADT approach is the integrated view of interoperability. In contrast, extant research is mainly concerned with various aspects of interoperability. For example, the EU-sponsored project ENSEMBLE has collected a large number of research contributions on enterprise interoperability. The following twelve themes have been identified (ENSEMBLE, 2012)

- Data Interoperability: "the ability of data (including documents, multimedia content and digital resources) to be universally accessible, reusable and comprehensible by all transaction parties." (ibid., p. 50).

- Process Interoperability: "The ability to align business processes of different entities (enterprises), in order to conduct business in a seamless way." (ibid., p. $58)$.

- Rules Interoperability: "The ability of entities to align and match their business and legal rules for conducting legitimate automated transactions that are also compatible with the internal business operation rules of each other." (ibid., p. 63).

- Objects Interoperability: "Objects interoperability refers to the networked interconnection of everyday objects. Devices or hardware components interoperability can be seen as a particular case of the object interoperability domain." (ibid., p. 66).

- Software Systems Interoperability: "Software Systems Interoperability refers to the ability of an enterprise system or a product to work with other enterprise systems or products." (ibid., p. 69).

- Cultural Interoperability: "The degree to which knowledge and information is anchored to a unified model of meaning across cultures." (ibid., p. 72).

\footnotetext{
${ }^{8}$ An extensive number of interoperability research references can be found in this document.
} 
- Knowledge Interoperability: "The ability of two or more different entities to share their intellectual assets, take immediate advantage of the mutual knowledge and utilise it, and to further extend them through cooperation." (ibid., p. 75).

- Services Interoperability: "The ability of an entity to seamlessly and automatically discover, aggregate and utilise a service that belongs to another entity." (ibid., p. 79).

- Social Networks Interoperability: "The ability of enterprises to utilise social networks for collaborations and interconnection purposes, by aligning their internal structure to the fundamental aspects of the social networks." (ibid., p. 83).

- Electronic Identity Interoperability: "The ability of different eID systems to collaborate in order to automatically authenticate entities and to pass on security roles and permissions to eID holders, regardless the system that they originate from." (ibid., p. 86).

- Cloud Interoperability: "The ability of cloud services to be able to work together with both different cloud services and providers, and other applications or platforms that are not cloud dependent." (ibid., p. 90).

- Ecosystems Interoperability: "The ability of instant and seamless collaboration between different ecosystems, ecosystems and independent entities, entities within the ecosystems and the ability of different independent entities to formulate virtual structures for specific purposes. (ibid., p. 93).

It is beyond the scope of this paper to do an extensive analysis of how the ADTapproach relates to these different themes. However, some observations can be made. First, all themes are about a specific aspect of interoperability, but it is not obvious how these themes are related to each other. Second, many themes seem to overlap, for example, cultural- and knowledge interoperability, which are both about knowledge. Third, some aspects are human and social in nature (cultural; knowledge; social networks; ecosystems), while other are of a more technical nature (software systems; objects; services; cloud). Still other are more related to the enterprise (data; process; rules; eID). The "granularity" of the themes is also varied; from electronic identity to culture and ecosystems. Finally, some categories appear hard to operationalize since they are so vaguely defined (culture; knowledge; ecosystem). Overall, a fragmented and diverse picture of interoperability is conveyed.

From the ADT-perspective, some of the themes can be directly related to the activity modalities. Data (and possibly objects) interoperability is associated with the spatialization modality; process interoperability with temporalization; and rules interoperability with stabilization. Social and human aspects are associated with the activity domain as a whole, since the nature of the domain is the determinant factor for knowledge, meaning, culture, etc. The technical aspects concern various capabilities needed to support interoperability between the activity domains.

Thus, there is clearly a potential for the activity domain construct to become a UoA towards which various aspects of interoperability can be grounded. However, the elaboration of this potential is a matter for further research. 


\section{CONCLUSIONS}

The position taken in this contribution is that sustainability requires that social and human aspects are included in interoperability investigations. The simple reason is that this is where we find the most challenging, and less attended problems in implementing interoperability. To this end, the Activity Domain Theory provides a "guiding lens" for elaborating concrete, practical implementation approaches, taking into consideration the constraints and enablers of socially organized work. Thus, this theory may be an alternative framework for advancing the understanding of sustainable interoperability between ESs.

\section{REFERENCES}

Alter, S. (2006). The Work System Method: Connecting People, Processes, and IT for Business Results. Larkspur, CA: Work System Press.

Argyres, N. S. (1999). The Impact of Information Technology on Coordination: Evidence from the B-2 "Stealth" Bomber. Organization Science, 10(2), 162180.

Bechky, B. A. (2003). Sharing meaning across occupational communities: The transformation of understanding on a production floor. Organization Science 14(3), 312-330.

Bechky, B. A. (2006). Gaffers, gofers, and grips: Role-based coordination in temporary organizations. Organization Science 17(1), 3-21.

ENSEMBLE (2012). Deliverable D2.1, EISB State of Play Report. Retrieved July $10^{\text {th }}, 2012$, from http://www.fines-cluster.eu/fines/jm/docman/Downloaddocument/119-ENSEMBLE_D2.1_EISB_State_of_Play_Report-v1.00.html

Grant, R. M. (1996). Toward a Knowledge-Based Theory of the Firm. Strategic Management Journal, 17, Winter Special Issue, 109-122.

Hoskin, K. (2004). Spacing, Timing and the Invention of Management. Organization, 11(6), 743 - 757.

IEEE (1990). Institute of Electrical and Electronics Engineers. IEEE Standard Computer Dictionary: A Compilation of IEEE Standard Computer Glossaries. New York, NY: 1990

Kaptelinin, V., \& Nardi, B. (2006). Acting with Technology - Activity Theory and Interaction Design. Cambridge, MA: The MIT Press.

Kangassalo, H. (1999). Are Global Understanding, Communication, and Information Management in Information Systems Possible? In P.P. Chen et al. (Eds.) Conceptual Modeling, LNCS 1565 (pp. 105-122).

Kim, J., Lee, Z., \& Gosain, S. (2005). Impediments to successful ERP implementation process. Business Process Management Journal, 11 (2), 158170.

King, B. K., Felin, T., \& Whetten, D. A. (2010). Finding the Organization in Organizational Theory: A Meta-Theory of the Organization as a Social Actor. Organization Science, 21(1), 290-305. 
Larsson, R. (1990). Coordination of Action in Mergers and Acquisitions Interpretative and Systems Approaches towards Synergy. Dissertation No. 10, Lund Studies in Economics and Management, The Institute of Economic Research, Lund: Lund University Press.

Levinthal, D. A., \& Warglien, M. (1999). Landscape Design: Designing for Local Action in Complex Worlds. Organization Science 10(3), 342-357.

Malone, T., Crowston, K. (1994). The Interdisciplinary Study of Coordination. ACM Computing Services, 26(1), 87-119.

Morgeson, F. P., \& Hofmann, D. A. (1999). The Structure and Function of Collective Constructs: Implications for Multilevel Research and Theory Development. Academy of Management Review, 24(2), 249-265.

Nickerson, J. A., \& Zenger, T. R. (2002). Being Efficiently Fickle: A Dynamic Theory of Organizational Choice. Organization Science, 13(5), 547-566.

Nicolini, D. (2011). "Practice as the Site of Knowing: Insights from the Field of Telemedicine," Organization Science, 22(3), 602-620.

Nonaka, I., \& von Krogh, G. (2009). Tacit Knowledge and Knowledge Conversion: Controversy and Advancement in Organizational Knowledge Creation Theory. Organization Science, 20(3), 635-652.

Schoonhoven, C. B., Meyer, A. D., \& Walsh J. P.(2005). Pushing Back the Frontiers of Organization Science. Organization Science, 16(4), 327-331

Sosa, M. E. (2011).Where Do Creative Interactions Come From? The Role of Tie Content and Social Networks. Organization Science, 22(1), 1-21.

Taxén, L. (2009). Using Activity Domain Theory for Managing Complex Systems. Information Science Reference. Hershey PA: Information Science Reference (IGI Global). ISBN: 978-1-60566-192-6.

Taxén, L. (2011). Modeling the Intellect from a Coordination Perspective. In B. Igelnik (Ed.), Computational Modeling and Simulation of Intellect: Current State and Future Perspectives. Hershey PA: IGI Global. ISBN: 978-1-60960$551-3$

Taxén, L., \& Lilliesköld, J. (2008). Images as action instruments in complex projects, International Journal of Project Management, 26(5), 527-536

Volkoff, O., Strong, D. M., \& Elmes, M. B. (2007). Technological Embeddedness and Organizational Change. Organization Science, 18(5), 832-848.

Wittgenstein, L. (1953). Philosophical investigations, Oxford: Blackwell.

Zinchenko, V. (1996). Developing Activity Theory: The Zone of Proximal Development and Beyond. In B. Nardi (Ed.) Context and Consciousness, Activity Theory and Human-Computer Interaction (pp. 283-324). Cambridge, Massachusetts: MIT Press. 